\title{
Structure-function relationship of bifunctional XynA
}

\author{
Wei Xie ${ }^{1}$, Qi Yu ${ }^{1}$, Ruiqin Zhang ${ }^{1}$, Yun Liu ${ }^{1}$, Ruoting Cao ${ }^{1}$, Sidi Wang ${ }^{1}$, Ruoting Zhan ${ }^{1}$, \\ Zhongqiu Liu ${ }^{1}$, kui wang ${ }^{2}$, and Caiyan $\mathrm{Wang}^{2}$ \\ ${ }^{1}$ Guangzhou University of Traditional Chinese Medicine \\ ${ }^{2}$ Guangzhou University of Chinese Medicine
}

January 3, 2021

\begin{abstract}
Xylan and cellulose are the two major constituents in numerous types of lignocellulose. Thus, bifunctional enzyme incorporated xylanase/cellulase activity has attracted considerable attention since it has great cost savings potential. Recently, a novel GH10 family enzyme XynA identified from Bacillus sp. was found to degrade both cellulose and xylan. To understand its molecular catalytic mechanism, here we first solve the crystal structure of XynA at $2.3 \AA$. XynA is characterized with a classic $(\alpha / \beta) 8$ TIM-barrel fold (GH10 domain) flanked by the flexible N-terminal domain and C-terminal domain. XynA has a longer N-terminal and C-terminal than most other GH10 family enzymes. The important thing is that the activity of our N-terminal truncated XynA_AN37 is significantly improved. And we found that the C-terminus is crucial to protein expression in solution. Protein thermal shift and enzyme activity assays reveal that conserved residues Glu182 and Glu280 are both important for catalytic activities of XynA, which is verified by the crystal structure of XynA with double mutant E182A/E280A. Molecular docking studies of XynA with xylohexaose and cellohexaose, together with site-directed mutagenesis and enzyme activity assay, demonstrate that Gln250 and His252 are indispensable to bifunctional activity. These results elucidate the structural and biochemical features of XynA, providing clues for further modification of XynA for industrial application.
\end{abstract}

\section{Structure-function relationship of b ifunctional XynA}

Wei Xie ${ }^{1}$, Qi Yu ${ }^{1}$, Ruiqing Zhang ${ }^{2}$, Yun $\mathrm{Liu}^{2}$, Ruoting $\mathrm{Cao}^{2}$, Sidi Wang ${ }^{3}$, Ruoting Zhan ${ }^{2}$, Zhongqiu Liu ${ }^{1}$, Kui Wang ${ }^{2 *}$, Caiyan Wang ${ }^{1 *}$

${ }^{1}$ Guangdong Key Laboratory for Translational Cancer Research of Chinese Medicine, Joint Laboratory for Translational Cancer Research of Chinese Medicine of the Ministry of Education of the People's Republic of China, International Institute for Translational Chinese Medicine, Guangzhou University of Chinese Medicine, Guangzhou, Guangdong, 510006, China.

${ }^{2}$ Research Center of Chinese Herbal Resource Science and Engineering, Key Laboratory of Chinese Medicinal Resource fromLingnan (Guangzhou University of Chinese Medicine), Ministry of Education, Joint Laboratory of National Engineering Research Center for the Pharmaceutics of Traditional Chinese Medicines, Guangzhou University of Chinese Medicine, Guangzhou, Guangdong, 510006, China. ${ }^{3}$ College of Fundamental Medical Sciences, Guangzhou University of Chinese Medicine, Guangzhou 510006, China.

\section{${ }^{*}$ Corresponding authors:}

Kui Wang,

Mailing address: Research Center of Chinese Herbal Resource Science and Engineering, Guangzhou University of Chinese Medicine, Guangzhou Higher Education Mega Center, No.232 Outer Ring East Rd., Panyu District, Guangzhou 510006, Guangdong Province, China.

Phone: 86-020-39358066; Fax: 86-020-39358066; 
E-mail: kuiwang@gzucm.edu.cn.

Caiyan Wang,

Guangdong Key Laboratory for Translational Cancer Research of Chinese Medicine, Joint Laboratory for Translational Cancer Research of Chinese Medicine of the Ministry of Education of the People's Republic of China, International Institute for Translational Chinese Medicine, Guangzhou University of Chinese Medicine, Guangzhou, Guangdong, 510006, China. Tel.: 86-020-39358875; Fax: 86-020-39358071;

E-mail: wangcaiyan@gzucm.edu.cn.

Running title: Structural basis of bifunctional GH10 xylanase XynA

\section{Abstract}

Xylan and cellulose are the two major constituents in numerous types of lignocellulose. Thus, bifunctional enzyme incorporated xylanase/cellulase activity has attracted considerable attention since it has great cost savings potential. Recently, a novel GH10 family enzyme XynA identified from Bacillus sp. was found to degrade both cellulose and xylan. To understand its molecular catalytic mechanism, here we first solve the crystal structure of XynA at $2.3 \AA$. XynA is characterized with a classic $(\alpha / \beta)_{8}$ TIM-barrel fold (GH10 domain) flanked by the flexible $\mathrm{N}$-terminal domain and $\mathrm{C}$-terminal domain. XynA has a longer $\mathrm{N}$-terminal and C-terminal than most other GH10 family enzymes. The important thing is that the activity of our $\mathrm{N}$-terminal truncated XynA_ $\Delta \mathrm{N} 37$ is significantly improved. And we found that the C-terminus is crucial to protein expression in solution. Protein thermal shift and enzyme activity assays reveal that conserved residues Glu182 and Glu280 are both important for catalytic activities of XynA, which is verified by the crystal structure of XynA with double mutant E182A/E280A. Molecular docking studies of XynA with xylohexaose and cellohexaose, together with site-directed mutagenesis and enzyme activity assay, demonstrate that Gln250 and His252 are indispensable to bifunctional activity. These results elucidate the structural and biochemical features of XynA, providing clues for further modification of XynA for industrial application.

Keywords : XynA, GH10, bifunctional xylanase/cellulase, crystal structure, molecular mechanism

\section{Introduction}

With the continuous consumption of oil resources and the development of the global economy, developing cleaner and more economical energy is of utmost importance (Victor \& Leape, 2015). The sources of biomass energy are very rich, including forestry byproducts and crop straws, which are renewable energy sources (Isikgor \& Becer, 2015; Sims, Mabee, Saddler, \& Taylor, 2010). During production, the plant cell walls must be depolymerized, which requires synergistic action of cellulase and xylanase (Broeker et al., 2018; Keegstra, 2010; Long et al., 2018; Xiaoyun Su et al., 2013). In addition to biofuel production, xylanase is also applied in various industries, such as the food industry, feed industry, paper industry, and brewing industry (Dornez, Verjans, Arnaut, Delcour, \& Courtin, 2011; Ito et al., 2019; Kumar, Marin-Navarro, \& Shukla, 2016; Lisov et al., 2017).

The thermostable glycoside hydrolase (GH) family with bifunctional activity is more advantageous in degrading lignocellulose biomass, and thus has great development potential for industrial application. Among the GH family, the GH10 family and GH11 family contain a large number of xylanases (Lombard, Golaconda Ramulu, Drula, Coutinho, \& Henrissat, 2014). Compared with GH11 xylanases, GH10 xylanase has an extensive substrate scope (Biely, Singh, \& Puchart, 2016; Chakdar et al., 2016; Collins, Gerday, \& Feller, 2005).(13-15) Furthermore, some GH10 xylanases have been found to degrade other polysaccharides, such as konjac glucomannan and tamarind xyloglucan (Fredriksen et al., 2019). The GH10 domain of GH10 family xylanases is responsible for catalytic activity. The regions outside the GH10 domain (N-terminal or C-terminal) may affect the thermal stability of the protein (Mahanta, Bhardwaj, Kumar, Reddy, \& Ramakumar, 2015). Because the binding ability of some xylanase and the substrate is relatively weak, it is difficult to detect their binding, such as the GH10A xylanase from the Arctic mid-ocean ridge ventilation system (Fredriksen et al., 2019). The xylanase GH10A is capable of degrading wheat arabinoxylan (WAX) 
and tamarind xyloglucan and it is difficult to detect the binding ability (Fredriksen et al., 2019). The bifunctional enzymes with xylanase/cellulase activity have been reported such as CbXyn10C (5OFJ) (Chu et al., 2017), SlXyn10A (1E0X) (Ducros et al., 2000a), CbXyn10B (4L4O) (Zhang et al., 2016) and CfCex (Liu, Zhang, \& Xu, 2012). CbXyn10C, Xyl10A, and CbXyn10B all have the classic $(\alpha / \beta)_{8}$ TIM-barrel fold structure, but there is no article revealing the "switch" of the GH10 family of xylanases from single function to dual function.

In a previous study, a novel GH10 xylanase termed XynA was identified from Bacillus sp. (Wang et al., 2019). XynA could function at a wide range of $\mathrm{pH}$ (maintains more than $60 \%$ activity at $\mathrm{pH}$ 5.0-7.5) and temperature (maintains more than $65 \%$ activity at $45{ }^{\circ} \mathrm{C}$ to $80{ }^{\circ} \mathrm{C}$ ) (Wang et al., 2019). Interestingly, XynA was found to be a bifunctional xylanase/cellulase enzyme, exhibiting activities towards both xylan substrates and a variety of cellulose substrates, including cellobiose, cellohexaose, carboxymethyl cellulose, filter paper, Avicel (microcrystalline cellulose), $p$-nitrobenzene-cellobioside and $p$-nitrobenzene-glucopyranoside (Wang et al., 2019). In addition, XynA hydrolyzed corn stover pretreated with cellulase, showing an obvious synergistic effect (Wang et al., 2019). These unparalleled characteristics make XynA become an attractive candidate in biotechnology applications, such as bioenergy production and pulp processing (Dhiman et al., 2014; Dodd \& Cann, 2009; You et al., 2019). However, the xyanase activity of XynA is much stronger than its cellulase activity, which may raise some problems during application, for instance, the insufficient degradation of substrates and the quality control (Dodd \& Cann, 2009).

To provide useful guidelines for design of XynA for future industrial application, we investigated the catalytic molecular mechanism of XynA for two substrates xyanase and cellulase based on $2.3 \AA$ crystal structure. Firstly, we designed a series of truncation at both ends of the XynA, we found the bifunctional activity of truncation XynA_AN37 is significantly improved. Then, we designed function assays and revealed that the conserved residues Glu182 and Glu280 are crucial for the bifunctional activity of XynA, and the other two residues Gln250 and His252 are essential to bifunctional activity of XynA. These catalytic features providing clues for further structure modification of XynA for industrial application.

\section{Materials and Methods}

\section{Site-directed mutagenesis}

The GenBank of XynA is QCO69162. The target protein was preceded by a 6xHis tag. We designed primer pairs with mutation points. The primers of E182A were used to cause the plasmid pET28a-XynA to undergo PCR. Then, the PCR product was digested with Dpn I (Thermo Scientific, Waltham, United States) for 1.5 hours at $37^{\circ} \mathrm{C}$, and the product was heated at $80{ }^{\circ} \mathrm{C}$ for 5 minutes to denature. Finally, the product was transformed into competent DH5 $\alpha$ cells. The successfully sequenced mutants were transformed into BL21-CodonPlus (DE3) cells to express the protein. The mutant E280A needed to be mutated based on pET28a-E182A. XynA E182Q/E280Q had the same construction method as XynA E182A/E280A. The purification methods of various mutants and truncations of XynA are the same as XynA.

\section{Expression and purification of the wild-type and mutant XynA proteins}

The recombinant plasmid pET28a-XynA was transformed into BL21-Codonplus (DE3) competent cells, and then a single colony was selected and inoculated into $100 \mathrm{~mL}$ of $\mathrm{LB}$ medium containing $30 \mu \mathrm{g} / \mathrm{mL}$ kanamycin (Sangon Biotech, Shanghai, China) and $32 \mu \mathrm{g} / \mathrm{mL}$ chloramphenicol (Sangon Biotech, Shanghai, China) and cultured in a $37{ }^{\circ} \mathrm{C}$ constant temperature shaking table at $180 \mathrm{rpm}$ for 10 hours. When the $\mathrm{OD}_{600}$ reached 0.6-0.8, the E. coli had reached the logarithmic growth stage. Protein expression was then induced with 0.2 $\mathrm{mM}$ isopropyl- $\beta$-D-thiogalactopyranoside (IPTG, Sangon Biotech, Shanghai, China) at $25{ }^{\circ} \mathrm{C}$ on a constant temperature shaker for 12 hours. Finally, the samples were collected by centrifugation at $4000 \mathrm{rpm}$ for 20 minutes at $4{ }^{\circ} \mathrm{C}$ and stored at $-80{ }^{\circ} \mathrm{C}$ until use.

The collected E. coli was suspended in buffer A containing $40 \mathrm{mM}$ Tris- $\mathrm{HCl}$ at $\mathrm{pH} 8.0$ with $250 \mathrm{mM} \mathrm{NaCl}$ and $10 \mathrm{mM}$ imidazole at $4{ }^{\circ} \mathrm{C}$, and then $1 \mathrm{mM} \beta$-mercaptoethanol (Amresco, Guangzhou, China) and 1 mM PMSF (Sigma, Beijing, China) were added. The resuspended E. coli cells were passed through a 
low-temperature ultrahigh-pressure cell disrupter at $1200 \mathrm{psi}$, and the obtained lysate was centrifuged at $14000 \mathrm{rpm}$ for 60 minutes $\left(4^{\circ} \mathrm{C}\right)$. After centrifugation, portions of the supernatant and the precipitate were retained (Coomassie brilliant blue: running glue samples) (Laemmli, 1970). The supernatant was divided into two parts: one part was directly combined with nickel-nitrilotriacetic acid (Ni-NTA) affinity resin (Qiagen, China); the other part was heated in a $65{ }^{\circ} \mathrm{C}$ water bath for 60 minutes and then centrifuged at $14000 \mathrm{rpm}$ for 60 minutes. After the second centrifugation, a part of the supernatant and the precipitate were retained, and the supernatant was combined with Ni-NTA. The two supernatants above were combined with Ni-NTA for 45-60 minutes. The hybrid protein was removed by using buffer A ( $40 \mathrm{mM}$ Tris- $\mathrm{HCl}$ at $\mathrm{pH} 8.0$ with 250 $\mathrm{mM} \mathrm{NaCl}, 10 \mathrm{mM}$ imidazole at $\left.4{ }^{\circ} \mathrm{C}\right)$, and the target proteins were eluted by using buffer $\mathrm{B}(40 \mathrm{mM}$ Tris- $\mathrm{HCl}$ at pH 8.0 with $250 \mathrm{mM} \mathrm{NaCl}, 250 \mathrm{mM}$ imidazole at $4{ }^{\circ} \mathrm{C}$ ). XynA was purified in two ways, one of which was heating in a $65{ }^{\circ} \mathrm{C}$ water bath for 60 minutes and the second was using a Hi Trap QHP column $(1 \mathrm{~mL}$ GE Healthcare, United States). The protein is bound to the column at loading. The conditions are then changed, and the bound components elute separately. Purification was carried out on a $1 \mathrm{~mL}$ QHP column with a $\mathrm{NaCl}$ gradient, and the protein solution was injected into the equilibrium column at a flow rate of $0.5 \mathrm{~mL} /$ minute. A linear $\mathrm{NaCl}$ gradient elution of 50 to $250 \mathrm{mM}$ was used, and $2 \mathrm{~mL}$ fractions of the eluate were collected over the entire gradient length.

The state of XynA in solution was analyzed by a Superdex 200 (10/300) increase column (GE Healthcare, United States). First, the chromatographic column was balanced with a buffer containing $150 \mathrm{mM} \mathrm{NaCl}, 40$ $\mathrm{mM}$ Tris- $\mathrm{HCl}$ at pH 8.0 and $1 \mathrm{mM}$ dithiothreitol (DTT, Sangon Biotech, Shanghai, China). Second, XynA loading. Finally, the column was eluted with buffer solution, and $2 \mathrm{~mL}$ fractions of eluate was collected.

\section{Protein crystallization, data collection and structure determination}

The home-grown crystals were initially screened manually using a laboratory-made kit at room temperature using a 96-well plate with diffused vapor diffusion. A $0.8 \mu \mathrm{L}$ aliquot of XynA protein solution $(8.0 \mathrm{mg} / \mathrm{mL}$, $20 \mathrm{mM}$ Tris- $\mathrm{HCl} \mathrm{pH}$ 8.0, $287 \mathrm{mM} \mathrm{NaCl}$ ) was mixed with a $0.8 \mu \mathrm{L}$ reservoir solution and equilibrated with a $100 \mu \mathrm{L}$ reservoir solution. Twenty-four hours later, the initial XynA crystals were obtained in 28\% PEG600 (w/v), $0.1 \mathrm{M} \mathrm{CaCl}_{2}$ and 0.1 M MES pH 6.0. The optimized crystallization conditions were 24\% PEG600 (w/v), $0.2 \mathrm{M} \mathrm{CaCl}_{2}, 0.1 \mathrm{M}$ MES pH 6.0, crystal length reaches $2 \mathrm{~mm}$. 29\% PEG600 (w/v), $0.1 \mathrm{M} \mathrm{CaCl}_{2}, 0.1$ M MES pH 6.0 and $25 \%$ glycerol were used as refrigerating protectors. Individual crystals were installed on a nylon loop and immediately cooled with liquid nitrogen prior to data collection. The individual crystals are installed on a nylon loop and immediately cooled with liquid nitrogen. In Shanghai Synchrotron Radiation Facility (SSRF-BL19U1) diffraction data was collected through X-ray diffraction and finally processed using HKL2000 (Otwinowski \& Minor, 1997). We use PDB 1V0L as a model to obtain the initial model. Based on the initial model, we use COOT to accurately modify the structure, and then use CCP4 for refinement. Finally, repeat the above steps until the structural requirements are met (Emsley, Lohkamp, Scott, \& Cowtan, 2010; McCoy et al., 2007). XynA finally obtained a $2.3 \AA$ resolution crystal structure. The crystallization conditions of the XynA E182A/E280A were the same as those of XynA, and the structure of the XynA E182A/E280A was obtained with a resolution of $2.9 \AA$. Information about the protein crystal structure is provided inTable 1 . Conservation analysis of protein structure was carried out through the ConSurf Server website and PyMOL software was used to prepare images.

\section{Multiple residue sequence alignment}

We downloaded the residue sequences of the GH10 family of xylanases such as CbXyn10C and SlXyn10A from the GenBank protein database. Then, we compared the residue sequences of XynA, the residue sequence of the GH10 family of monofunctional xylanases, and the residue sequence of the GH10 family of bifunctional xylanases using ClustalW software and the ESPript3 website.

\section{Biolayer interferometry binding studies}

The ForteBio Octet@ RED96 interaction analyzer(39-41) (ForteBio, United States) was used to detect the binding of XynA to the oligosaccharide substrates (cellobiose, xylohexaose and cellohexaose) (Megazyme, Ireland) and xylan (BWX) (Shanghai, China) (Li, Tian, Wang, Zhu, \& Sun, 2017; Petersen, 2017). First, 
XynA was dialyzed against PBS. Second, the equivalent biotinylation kit (Biotinylation Kit, Jiangsu Bomeida Life Science Co., Ltd.) was added to bind XynA protein for 60 minutes for biotinylating. Finally, the unbound biotin was removed with a desalination column. Before the experiment, we wetted the biosensor (SSA, Shenzhen Baokeda Biotechnology Co., Ltd.) with PBS for 10 minutes. In the first step, we equilibrated the sensor with PBS for 2 minutes. In the second step, XynA was loaded onto the SSA sensor until it was saturated. Third, we equilibrated with PBS for 3 minutes. Fourth, diluted substrate solutions $(12.5,25,50$, $100,200$, and $250 \mu \mathrm{M})$ were sequentially added and dissociated from low concentration to high concentration, and each combination and dissociation time was 300 seconds. A sensor not bound to the biotinylated XynA was used as a baseline control for calibration. The whole process was carried out at room temperature, and all tests were repeated three times.

\section{Protein thermal shift assay}

SYPRO Orange Protein Gel Stain 5000× (Thermo Scientific, United States) was diluted with protein buffer solution $(20 \mathrm{mM}$ Tris- $\mathrm{HCl}$ at $\mathrm{pH} 8.0$ with $287 \mathrm{mM} \mathrm{NaCl})$ to $200 \times$. A $30 \mu$ g portion of protein $(5.0 \mathrm{mg} / \mathrm{mL})$ was mixed with BWX and incubated on ice for 60 minutes, then centrifuged at $14000 \mathrm{rpm} /$ minutes for 10 minutes. After centrifugation, the protein was evenly mixed with $2.5 \mu \mathrm{L} 200 \times$ SYPRO Orange, and the total volume was $20 \mu \mathrm{L}$. The mixture was heated from $25{ }^{\circ} \mathrm{C}$ to $99{ }^{\circ} \mathrm{C}$ at a rate of $0.2{ }^{\circ} \mathrm{C} /$ minute, and each sample was prepared in triplicate. An Applied Biosystems real-time PCR instrument (7500 Fast, Thermo Scientific, United States) was used to determine the protein denaturation temperature (exposure temperature of hydrophobic amino acid residues), and the results were generated using Origin software (Niesen, Berglund, \& Vedadi, 2007).

\section{Molecular docking}

First, the PDB files of XynA, xylohexaose and cellohexaose were prepared using the Protein Preparation Wizard of Maestro (Maestro11.9, Schrödinger, LLC, New York, 2019) to prepare the model structure for docking (Friesner et al., 2004; Sastry, 2013). Then, the appropriate docking pocket was selected, and the molecular docking was performed. The docking scores of each ligand were determined with the best posture on Maestro. The criterion for hydrogen bond judgment is that the distance between the acceptor and donor atoms should be less than $3 \AA$. Output the docking file through Maestro, and finally use PyMOLsoftware to make figure.

\section{Determination of the enzyme activity by DNS}

The DNS method was used to detect the xylanase activity of the XynA and XynA E182A/E280A proteins. In this study, BWX (Lot\# X4252, [?] 90\% purity) was used as the substrate, and xylose was used as the standard. The reducing sugars released from BWX were measured by DNS reagent to estimate the xylanase activity. The hydrolyzed product oligosaccharides (reducing sugars) undergo redox reactions with 3,5-dinitrosalicylic acid (DNS) to produce 3-amino-5-nitrosalicylic acid (brown red). First, we took the appropriate concentration $(25,250,500,1000 \mathrm{nM})$ of the protein and $0.5 \%(\mathrm{w} / \mathrm{v})$ of BWX and mixed them evenly in $100 \mathrm{mM}$ sodium citrate at $\mathrm{pH} 6.5$, and the total volume was $50 \mu \mathrm{L}$. Second, the reaction system was incubated at the optimal temperature of $65{ }^{\circ} \mathrm{C}$ for 10 minutes. Third, the DNS reagent was added to the reaction system, and the mixture was incubated at $95{ }^{\circ} \mathrm{C}$ for 5 minutes. Finally, the reaction system was cooled in an ice bath to room temperature and diluted with $100 \mathrm{mM}$ sodium citrate buffer solution at $\mathrm{pH}$ 6.5 , and the absorbance was determined at $540 \mathrm{~nm}$ with an enzyme label analyzer.

\section{Results}

\section{The overall structure of XynA}

The full-length XynA protein was expressed and purified with removal of nucleic acids by anion exchange column QHP. XynA contains 408 residues and the molecular weight of the tagged full-length protein was $49.8 \mathrm{kDa}$ with an isoelectric point of 6.64 . It was identified as one monomer in the solution by gel filtration (Figure 1A). We found that xylanase 10A (SIXyn10A, PDB code 1V0L) fromS. lividans has the highest sequence similarity (30\%) with XynA by using Phyre2 server (Gloster et al., 2004; Kelley, Mezulis, Yates, 
Wass, \& Sternberg, 2015). We used this structure as a search model to solve the crystal structure of XynA by molecular replacement method.

The determined $2.3 \AA$ crystal structure of XynA (residues 22-393) with a $R_{\text {free }}$ of 0.245 (Table 1 ). The space group is $P$ 4122 , and one molecule was present in the asymmetric unit. In the refined model, residues Leu52, Ala53, Asp359, Ala360, Asn361, Gly378, and Glu379 are missing due to disordered configuration. The XynA structure is composed of $12 \alpha$-helices and $14 \beta$-sheets, among which $\alpha 2$ - $\alpha 11$ helices and $\beta 3-\beta 10$ sheets form a classical $(\alpha / \beta)_{8}$ TIM-barrel fold(Figure 1B) . Overall, the mug-shaped XynA is comprised of an N-terminal domain (residues 1-67), GH10 domain (residues 68-326) and C-terminal domain (residues 327-408) (Figure 1C) . The body of "mug" is GH10 domain, which has $3 \alpha$-helices separated by loop regions, named $\alpha 2, \alpha 4$, and $\alpha 7$ (Figure 1B). These three $\alpha$-helices are not affecting the overall conformation of classical $(\alpha / \beta)_{8}$ TIM-barrel fold.

\section{The thermostability and activity of N-terminal and C-terminal to XynA}

We compare the crystal structures of XynA with other GH10 family enzymes. Of note, only the GH10 domain is in the other protein structures mentioned above, the N-terminal and C-terminal domains are unique for XynA (Figure 2A ). The typical GH10 domain is well conserved in GH10 family, as evidenced by the low RMSD $(1.599 \AA)$ of structural superimposition of XynA, TsXy1A and SIXyn10A. In order to explore the effects of $\mathrm{N}$-terminal and $\mathrm{C}$-terminal on XynA, we constructed deleted residues 1-67 named XynA_$\Delta \mathrm{N}$ and deleted of residues 327-408 named XynA_ $\Delta$ C. Only XynA_ $\Delta$ N37 (delete residues 1-36) expresses soluble protein, XynA $\Delta \mathrm{C}$ has been unable to express soluble protein. We tested the activity and thermal stability of XynA_ $\Delta$ N37 protein. We found that the melting temperature of XynA_ $\Delta$ N37 is $71.32{ }^{\circ} \mathrm{C}$, which is 2.21 ${ }^{\circ} \mathrm{C}$ lower than that of XynA (Figure 2B ). XynA_ $\Delta \mathrm{N} 37$ has a significant increase in the hydrolytic activity of the three substrates BWX, WAX and barley $\beta$-glucan (Figure 2C ). Since $71.32{ }^{\circ} \mathrm{C}$ is a relatively high melting temperature in xylanase, the difference in melting temperature between XynA_ $\Delta \mathrm{N} 37$ and XynA can be ignored. We believe that XynA_ $\Delta$ N37 increases the activity while maintaining the original thermal stability. The N-terminal and C-terminal form the handle of this "mug" together. We find that the Nterminal residues Arg25, Asn29, Arg31, and Arg36 establish abundant hydrogen bond interactions with the residues Glu296, Arg300, Phe303, Ser304, His305, and Val308 at the body GH10 domain (Figure 2D ). Similarly, the C-terminal residues Val330, His332, Arg342, Tyr343 and Glu344 form extensive hydrogen bond interactions with residues His89, Glu296, Phe316, Trp317, and Ala318 of the GH10 domain (Figure 2E ). The residues participated in hydrogen bond interactions are all located in $\alpha$-helices and loops. The difference between XynA and other GH10 families exists in the N-terminal and C-terminal domain. The C-terminal residues (Glu338-Glu349) form $\alpha$-helix 12 , which makes the classical $(\alpha / \beta)_{8}$ TIM-barrel fold more complete. The N-terminal $(\alpha 1, \beta 1$ and $\beta 2)$ and C-terminal domain $(\alpha 12, \beta 11-\beta 14)$ is partially crossover, and there are two $\alpha$-helices and six $\beta$-sheets that are adjacent to the TIM barrel.

\section{Sequence and structure analyses of XynA with other GH10 xylanase}

To find the catalytic residues of XynA, we performed multiple sequence alignments of XynA with five GH10 family enzymes, namely CbXyn10C (C. bescii), SlXyn10A (S. lividans), BsXynA (Bacillus sp. ), TmXynB (T. maritima) and TsXy1A (T. saccharolyticum). Among them, XynA, CbXyn10C and SIXyn10A are xylanases with xylanase and cellulase activity. BsXynA, TmXynB, and TsXy1A are strict xylanase enzyme. We find that the residues in the GH10 domain, such as Phe90, Glu96, Lys100, Gly133 and Trp137 are much conserved(Figure 3). Though XynA and BsXynA both come fromBacillus sp., BsXynA is a traditional GH10 family xylanase that only catalyzes the degradation of xylan (Zhou et al., 2014a). The architecture of GH10 domain is similar in XynA and BsXynA, but the subtle sequence difference in fifteen residues indicates that these sites may determine the monofunction or bifunction (Figure 3 ). By structural superposition and sequence comparison among CbXyn10C, SIXyn10A, TmXynB, TsXy1A and XynA, we infer that Glu182 and Glu280 are putative catalytic residues of XynA (Chu et al., 2017; Han et al., 2013; Ihsanawati et al., 2005; V Ducros et al., 2000). Further analysis indicates that most residues related to monofunctional and bifunctional activity are conserved and overlapped, containing Glu96, Asn97, Lys100, His133 and Trp137. The residues specific to bifunctional activity include Tyr216, Asn217, Ile223, Trp257 and Tyr288. 


\section{The xylanase activity of XynA}

To explore the catalytic properties of XynA, we first investigated the optimal enzyme concentration and the xylanase activity of XynA. Using $0.5 \%$ (w/v) beechwood xylan (BWX) as substrate in $100 \mathrm{mM}$ sodium citrate buffer, $\mathrm{pH} 6.5$ at $65^{\circ} \mathrm{C}$ for 10 minutes, as the concentration of XynA protein increased, the percentage of degraded sugar increased until it reached a plateau (Figure 4A). Based on this reaction curve, $500 \mathrm{nM}$ is regarded as the optimal concentration for XynA.

The activity of GH10 family enzymes requires two conserved catalytic residues glutamate, and the mutation of glutamate will completely inactivate the protein (Derewenda et al., 1994). To verify the effect of these two glutamic acids on XynA activity, we double mutated Glu182 and Glu280 to alanine and produced the mutant XynA E182A/E280A protein. The xylanase activity of the mutants (XynA E182A/E280A) was determined using the DNS method. The results displayed that WT had the highest activity at a concentration of 500 $\mathrm{nM}$, while the E182A/E280A mutant is inactive, even at an extremely high concentration (1 mM) (Figure 4B) .

To analyze the binding mode and interaction patterns of XynA and its substrates, we have tried hard to prepare the crystals of XynA WT, E182A/E280A or E182Q/E280Q with cellobiose, cellohexaose, or xylohexaose complexes, respectively. However, all trials failed, only the apo crystals of WT and mutant XynA were obtained. This may be explained by the weak and difficult detection features for the GH10 family proteins and their substrates according to previous studies (Baumann et al., 2011). However, through Surface Plasmon Resonance (SPR) assay, we detected the binding affinity of BWX to both the WT and E182A/E280A mutant XynA(Figure 4C). The binding constants indicated that the binding ability of the WT to BWX was stronger than that of the E182A/E280A mutant. We found that WT does not bind to oligosaccharides by SPR assay, which is consistent with the fact that it is difficult for oligosaccharides to enter the crystal structure (Figure 4C) .

The protein thermal shift assay indicates that XynA is much thermostable, as evidenced by the Tm value $73.53{ }^{\circ} \mathrm{C}$. Moreover, the Tm value $75.5^{\circ} \mathrm{C}$ of XynA bound with BWX is higher than that of the apo protein, indicating that substrate binding could stabilize XynA(Figure 4D). Similarly, the Tm value of the mutant before and after the BWX binding also increased, indicating that the substrate binding also affects the stability of the mutant.

Crystal structure can provide direct hints to study the protein features and properties. To elucidate how E182/E280 affect the enzyme activity of XynA, we determined the crystal structure of XynA E182A/E280A at $2.9 \AA$. The overall architecture of XynA E182A/E280A is highly similar to the WT XynA, yielding a C $\alpha$ RMSD of $0.218 \AA$. A major difference observed between the WT and mutant is an angle swing in the Nand the C-terminus. We found that the previously established hydrogen bonds between Glu182 and Trp137, Asn181 and Gln250 as well as Glu280 and with Asn217 and His252 disappeared in the E182A/E280A mutant (Figure 4E, 4F) .

\section{Molecular docking of XynA with xylohexaose and cellohexaose and the mutant activity con- firmation}

Since the cocrystal structure of XynA complexed with substrates is unsuccessful, to investigate the catalytic mechanism of XynA, we performed molecular docking studies. The sugar chains of xylan and cellulose are too long to suitable for molecular docking, therefore, xylohexaose and cellohexaose are substrates for docking. Viewing the putative binding mode, these two substrates situated in the center of the mouth of the XynA "mug" structure (Figure 5A, 5B). Xylohexaose formed ten hydrogen bonds with surrounding residues in the active pocket (mug mouth), including Asp141, Asn143, Glu96, Lys100, Trp314, Asn220 and Ser223. Cellohexaose formed eleven hydrogen bond interactions with surrounding residues, such as Asp74, Glu96, Glu98, Try102, Glu182, Gln250, His252 and Asn220. Among the residues in putative substrate binding pocket, only five residues are varied between bifunctional enzymes XynA and CbXyn10C, namely 140, 216, 223, 257 and 288, indicating conservation in sequence and structure (Figure 5F ). The centric Glu182 and Glu280 are surrounded by Glu96, Asn97, Lys100, Tyr216 and Asn217, forming a pocket embracing the 
sugar chains of substrate. Based on molecular docking results, residues Gln250 and His252 are speculated with relation to the bifunctionality of XynA. According to the results, Asp74, Glu96, Gln250, His252 and Asn220 formed hydrogen bond with cellohexaose. Based on the classification of residues, we designed mutants D74A, E96A, Q250A, Q250E, H252A and N220E. In light of the residues of CbXyn10C interacting with the substrate, Val216 and Ala254 are potential residues that could interact with the substrate in the XynA sequence (Chu et al., 2017). We designed mutants N97E, V216E and A254E. We used WAX, BWX and barley $\beta$-glucan as substrates, and the activities of these mutants relative to that of the XynA (abbreviated as WT in Figure 5 ) was determined by the 3,5-dinitrosalicylic acid (DNS) method (Figure 5C, 5D, $\mathbf{5 E}$ ). We found that the four mutants (E182A/E280A, E182Q/E280Q, Q250A, and H252A) were inactive towards three substrates. The mutants E96A and N97E lost their activity towards BWX and were unable to degrade WAX and barley $\beta$-glucan by more than $50 \%$. The mutants V216E, N220E, and A254E showed significantly reduced degradation activity on WAX, BWX, and barley $\beta$-glucan. The mutant Q250E showed reduced degradation activity on WAX and BWX and no activity against barley $\beta$-glucan. The mutant D74A showed significantly weaker degradation activities against WAX and barley $\beta$-glucan. E182A/E280A and E182Q/E280Q were completely inactive against these three substrates, further confirming that Glu182 and Glu280 were the key catalytic residue residues.

\section{Discussion}

The most important features of XynA are its thermal stability, bifunctionality and low homology. It has been reported that its half-lives at $65{ }^{\circ} \mathrm{C}$ and $70{ }^{\circ} \mathrm{C}$ are 12 hours and 1.5 hours, respectively (Wang et al., 2019). We can take advantage of the thermal stability of this protein. We tried to purify the protein by heating it in a water bath at $65{ }^{\circ} \mathrm{C}$ for 1 hour, which may affect the structure of the protein, so we also used an anion-exchange column QHP for purification. During the crystallization process, we found that the protein purified by the QHP column crystallized quickly, and the crystal morphology was regular, while the protein purified by heating crystallized slowly, and the crystal morphology was irregular. These two kinds of crystallization indicate that the high temperature influences the conformation and stability of the protein, resulting in differences in the crystallization.

In addition to the typical $(\alpha / \beta)_{8}$ TIM-barrel fold GH10 domain structure, XynA has N-terminal and Cterminal. By homology analysis of the overall structure of XynA, the homology of the "mug handle" formed by the N-terminal and C-terminal parts of XynA is low, the $\beta$-sheet conservatism inside the "mug" is high, and the outer part of the $\alpha$-helix outside the "mug" is not conserved, while the inner part of the $\alpha$-helix is very conserved (Figure 5F).It has been reported that the N-terminus and C-terminus might affect the thermal stability, activity and substrate specificity of the protein (Mahanta et al., 2015; Song, Tsang, \& Sylvestre, 2015; Wang et al., 2019; Zheng et al., 2016). By truncating the N-terminal $\alpha$ helix (1-36), we proved that the N-terminal did not affect the thermal stability of XynA, and truncating the N-terminal alpha helix enhanced the activity of XynA (Figure 2B, 2C) . In the structure, we see that the N-terminal alpha helix (1-36) interacts with the GH10 domain by hydrogen bonding (Figure 2D). This hydrogen bond restricts the $(\alpha / \beta) 8$ TIM-barrel fold and cannot swing better to accommodate the substrate and promote its hydrolysis. We tried many C-terminal truncations, but none of them were soluble. This proves that the presence of the C-terminus is essential for protein expression. Our analysis showed that the inside of the "mug" is bound to the substrate and degrades the substrate, so the residues are more conserved.

We found and verified two key residues Glu182 and Glu280 by structural analysis of XynA. In the catalytic process, Glu182 and Glu280 are catalytic nucleophiles/bases and catalytic proton donors, respectively (Withers et al., 1986). Comparing the properties of WT with those of the double mutant showed the activities, structures and stabilities were all different, proving the importance of Glu182 and Glu280. Glu182 and Glu280 formed hydrogen bonds with surrounding residues could "lock" the substrate to be catalyzed after entering the active pocket. The hydrogen bonds formed by the side chains of Glu182 and Glu280 with surrounding residues account for the majority interaction. Since the residues 182 and 280 are located in the loop area, the reduced interaction also makes this part more flexible, thereby reducing the binding affinity to substrate. This is in consistent with the SPR result. At the same time, WT has the ability to hydrolyze 
the substrate, while the E182A/E280A has no hydrolysis ability, which also shows that the mutation affects the hydrolysis activity by affecting the binding of the substrate. We attempted to determine the cocrystal structure of the proteins (WT, E182A/E280A, and E182Q/E280Q) with substrates xylohexaose and cellohexaose to reveal the specific catalytic mechanism. However, neither the incubation of the protein with the substrate nor the immersion of the crystal in the substrate successfully afforded cocrystals. From the SPR results, we also observed that XynA does not bind to xylohexaose and cellohexaose. The substrate cannot be immobilized in the protein, so it is difficult to obtain a cocrystal structure.

We obtained the simulated structures of XynA and xylohexaose and of XynA and cellohexaose by molecular docking. The glucose unit has one more $-\mathrm{CH}_{2} \mathrm{OH}$ group than the xylose unit in its molecular structure. Therefore, cellohexaose requires more space than xylohexaose to accommodate the sugar chains. It is found that the XynA active pocket can hold xylohexaose and cellohexaose. This shows that XynA can accommodate sugar chains of different substituents and thus exhibit a broad substrate scope. Compared with the residues that formed hydrogen bonds with xylohexaose and cellohexaose, Asp74, Glu182, Gln250 and His252 are unique residues that form hydrogen bonds between XynA and cellohexaose. According to the molecular docking, Glu96, Lys100, Gln250 and His252 are particularly conserved in the residue sequences of the GH10 family and interact with substrates in CbXyn10C and SlXyn10A.

We find that four mutants, E182A/E280A, E182Q/E280Q, Q250A, and H252A are all inactive towards three substrates. We are not surprised that Glu182 and Glu280 are required for activity, as our results show that they are key catalytic residues. After being replaced with alanine, the activities for the three substrates completely disappeared, which proves that the two residues His252 and Gln250 also related to catalysis. Our analysis reveals the conservation of residue His252 and Gln250 in the GH10 family and the uniformity of its interactions with substrates (Figure 3 ). The mutant H252A and Q250A only reduce the activity of the CbXyn10C protein (Chu et al., 2017). However, H252A and Q250A of XynA lose its activity towards the three substrates, proving that His252 and Gln250 are important residues in XynA. For most of the xylanases in the GH10 family, the catalytic residues are Glu residue (Chu et al., 2017). But we find that in addition to Glu182 and Glu280 as catalytic residues, XynA also has the two residues His252 and Gln250. From the crystal structure, it is found that Glu280 is located in the loop region (more flexible). The side chain residues of His252 form hydrogen bonds with Glu280 side chains, and the main chain residues of Gln250 form hydrogen bonds with Glu280 main chain residues. The hydrogen bonding interaction makes the structure of Glu280 more stable, so that the side chain is closer to the substrate, thereby playing the role of electron transfer. The mutation of His252 prevents Glu280 from getting close to the substrate, making it difficult to carry out electron transfer and making the protein inactive (Figure 6). Glu182 is also located in the loop area, which is easier to swing and difficult to bind to the substrate. The side chain residues of Gln250 and Glu182 side chain residues form hydrogen bonds, which make the structure of Glu182 more stable and play the role of electron transfer (Figure 6). The mutation of Gln250 prevents Glu182 from getting close to the substrate, making it difficult to perform electron transfer. Therefore, we believe that a hydrogen bond network is formed around Gln250 to mediate the effects of Glu182 and Glu280.

The mutants D74A, E96A, N97E, N220E, V216E and A254E all have reduced activity to various extents, proving that these sites all contribute to the activity of the substrate. We indicate that the residues that interact with Glu182 including Trp137, Asn181, Asn217 and Gln250 and the residues that interact with Glu280 including His133, Asn217, Gln250 and His252 are very conserved in CbXyn10C, and these residues interact with the substrate, fully reflecting the unity of the GH10 family. Finally, we propose the catalytic mechanism of XynA. In the first glycosylation step, E280 acts as a nucleophile (since Glu280 has less interaction with surrounding residues than Glu182, Glu280 is more flexible to generate electron transfer first), attacking the anomeric center to displace glycosides and form glycosylase intermediates. At the same time, Glu182 acts as an acid catalyst and protonates the glycoside oxygen when the bond is broken. In the second deglycosylation step, the glycosylase is hydrolyzed by water, and Glu182 acts as a base catalyst, deprotonating the water molecules when it attacks. As the catalysis progresses, Gln250 and His252 interact with the catalytic residue to bring the catalytic residue close to the substrate. At the same time, Gln250 and His252 interact with the substrate to affect the catalysis (Figure 6) . 
XynA is a heat-stable and acid-stable xylanase in the GH10 family, and Celluclast 1.5L can synergistically hydrolyze pretreated corn stover. These properties indicate that it may be effective in food, animal feed and biofuel production. Analysis of the XynA crystal structure can provide a basis for its transformation, making it more suitable for commercial applications such as biofuel production.

\section{Data availability}

The atomic coordinates and structure factors of xylanase XynA structure has been uploaded to the Protein Data Bank (XynA PDB code: 7D88 and XynA E182A/E280A PDB code: 7D89). All remaining data are included in the article.

\section{Acknowledgements}

This work was supported by the following grants: the Youth Scientific Research Training Project of GZUCM (No. 2019QNPY07), the National Natural Science Foundation of China (No. 31700625), Guangdong Key Laboratory for Translational Cancer Research of Chinese Medicine (No. 2018B030322011), the National Natural Science Foundation of China (31802099), the Pearl River Talent Recruitment Program of Guangdong Province (2017GC010372), the Natural Science of Foundation of Guangdong Province, China (2018A030310497), the Research Program for Young Innovative Talents in Higher Education Institutions of Guangdong Province (2017KQNCX037) and Shanghai Synchrotron Radiation Facility.

\section{Conflict of Interest}

The authors declare no conflict of interest.

\section{Author contributions}

Caiyan Wang, Kui Wang and Zhongqiu Liu: Conceptualization, Methodology.

Wei Xie: Data curation, Writing- Original draft preparation.

Wei Xie, Qi Yu, Ruiqing Zhang, Yun Liu, Ruoting Cao, Sidi Wang and Ruoting Zhan: Software.

Wei Xie, Qi Yu, Caiyan Wang and Kui Wang: Visualization, Investigation.

Caiyan Wang, Kui Wang and Zhongqiu Liu: Writing-Reviewing and Editing.

Table 1 . Data collection and refinement statistics.

\begin{tabular}{|c|c|c|}
\hline & XynA (7D88) & XynA E182A/E280A (7D89) \\
\hline \multicolumn{3}{|l|}{ Data collection } \\
\hline Wavelength $(\AA)$ & SSRF-BL19U1 & SSRF-BL19U1 \\
\hline Space group & $P 4122$ & $P 4122$ \\
\hline $\mathrm{a}, \mathrm{b}, \mathrm{c}(\AA)$ & $109.842,109.842,85.57$ & $110.193,110.193,84.983$ \\
\hline$\alpha, \beta, \gamma\left(^{\circ}\right)$ & $90.00,90.00,90.00$ & $90.00,90.00,90.00$ \\
\hline Resolution $(\AA)$ & $15.29-2.345(2.429-2.345)^{\mathrm{a}}$ & $49.28-2.894(2.997-2.894)^{\mathrm{a}}$ \\
\hline$R_{\text {merge }} \mathrm{b}$ & $0.158(1.074)$ & $0.108(0.550)$ \\
\hline $\mathrm{I} / \sigma_{(\mathrm{I})}$ & $30(2.6)$ & $34(5.83)$ \\
\hline Completeness (\%) & $99.35(98.73)$ & $99.56(96.27)$ \\
\hline Redundancy & $25.3(23.6)$ & $25.5(27.1)$ \\
\hline \multicolumn{3}{|l|}{ Refinement } \\
\hline Resolution $(\AA)$ & $15.29-2.345$ & $49.28-2.894$ \\
\hline No. reflections & $22400(2182)$ & $12123(1135)$ \\
\hline $\mathrm{R}_{\text {work }}{ }^{c} / \mathrm{R}_{\text {free }}{ }^{\mathrm{d}}$ & $0.2213 / 0.2446$ & $0.2378 / 0.2690$ \\
\hline No. atoms & 3041 & 2884 \\
\hline Protein & 2972 & 2856 \\
\hline Ligand & 2 & 2 \\
\hline
\end{tabular}




\begin{tabular}{lll}
\hline & XynA (7D88) & XynA E182A/E280A (7D89) \\
\hline Water molecules & 67 & 26 \\
B-factors $\left(\AA^{2}\right)$ & & \\
Protein & 55.49 & 63.42 \\
Ligand & 51.66 & 49.77 \\
Water molecules & 56.34 & 47.08 \\
Bond lengths ( $\AA)$ & 0.009 & 0.014 \\
Bond angles (ọ) & 1.08 & 1.53 \\
Ramachandran favored (\%) & 95.51 & 96.21 \\
allowed & 3.93 & 3.21 \\
Outliers $(\%)$ & 0.56 & 0.58 \\
\hline
\end{tabular}

${ }^{a}$ Values in parentheses are for the highest-resolution shell. ${ }^{\mathrm{b}} \mathrm{R}_{\text {merge }}=\Sigma|(\mathrm{I}-<\mathrm{I}>)| / \sigma(\mathrm{I})$, where $\mathrm{I}$ is the observed intensity. ${ }^{c} R_{\text {work }}=\Sigma_{\text {hkl }}|| \mathrm{Fo}|-| \mathrm{Fc}|| / \Sigma_{\mathrm{hkl}}|\mathrm{Fo}|$, calculated from working data set. ${ }^{\mathrm{d}} \mathrm{R}_{\text {free }}$ is calculated from $5.0 \%$ of data randomly chosen and not included in refinement.

Figures and Figure legends
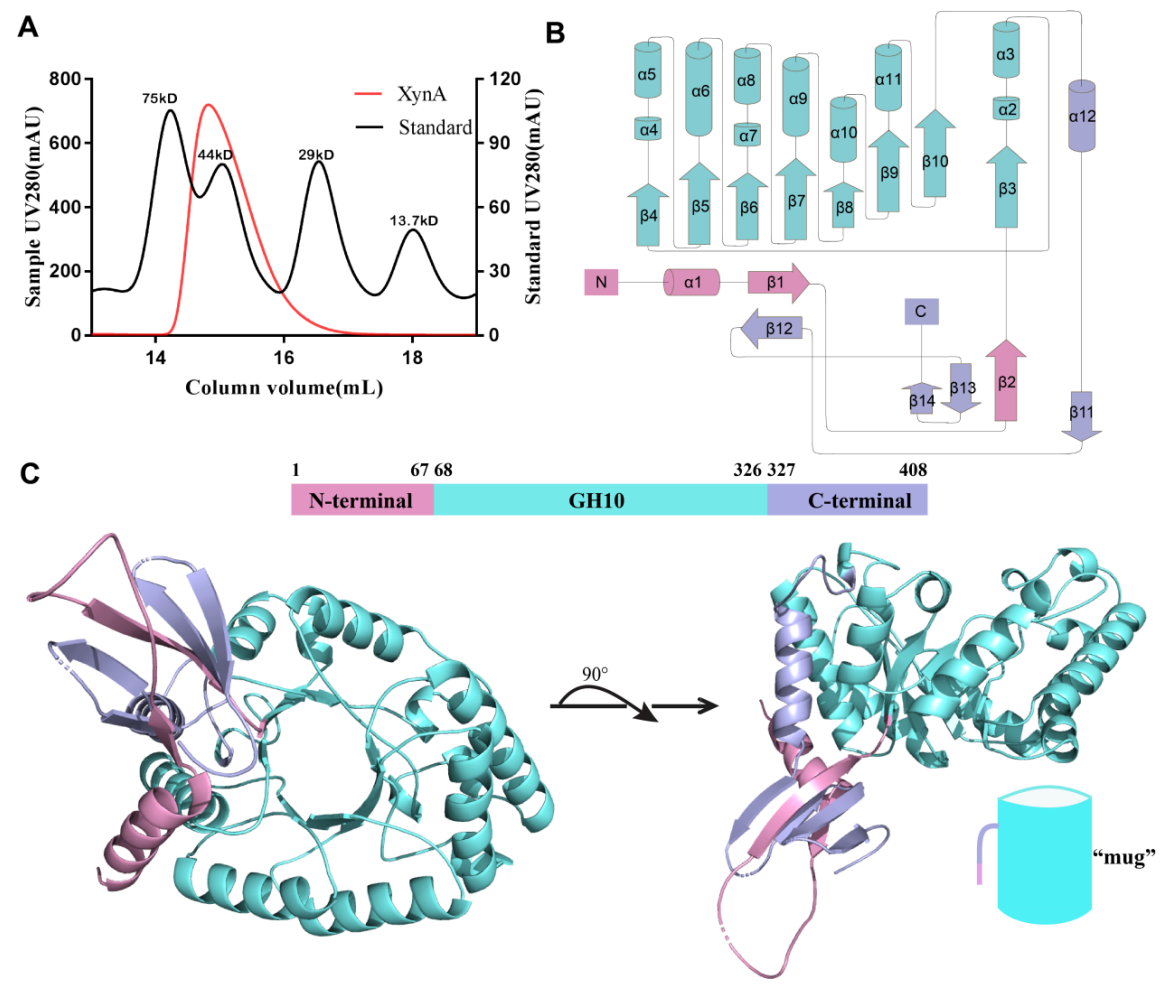

Figure 1. XynA protein and its structure. (A) Gel-filtration analysis showing XynA as monomer in solution. The red and black lines represent the UV absorption of XynA and the standard proteins at 280 nM respectively. (B) The topological organization of XynA. The GH10 catalytic domain, the N-terminal domain and C-terminal domain are colored in cyan, pink and purple, respectively. (C) Overall structure of $\mathrm{XynA}$ is like a shape of "mug". The color is assigned as shown in front. 

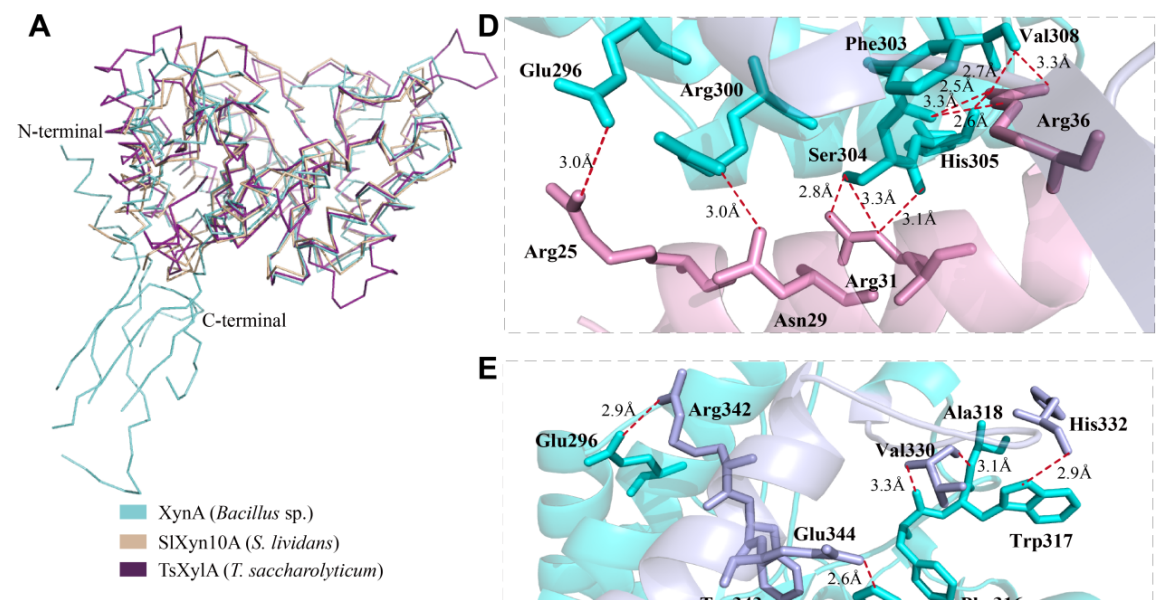

E
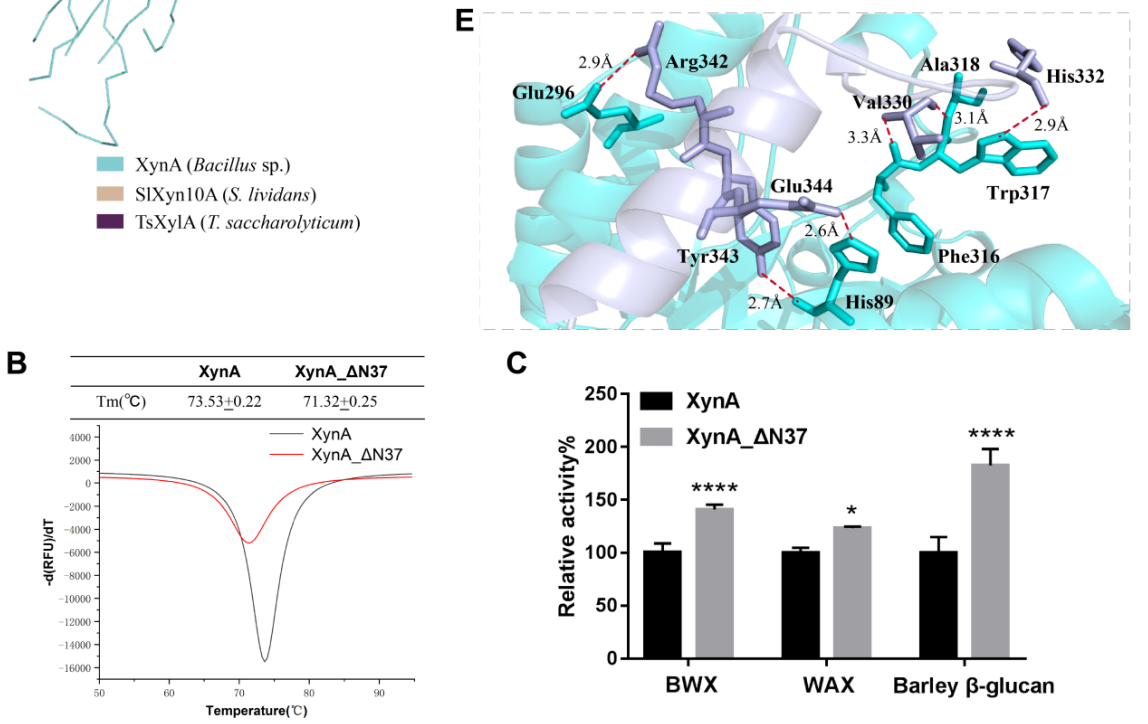

C

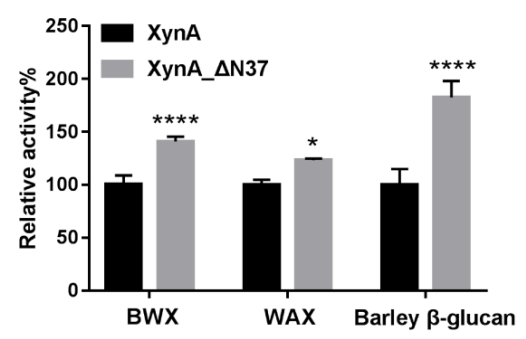

Figure 2. The analysis of N-terminal and C-terminal to XynA. (A) Structural superposition of XynA, SlXyn10A (PDB code 1V0L), and TsXy1A (PDB code 3W25). (B) Protein thermal shift assay of XynA and XynA $\_$N37. (C) Relative activities of the XynA $\Delta$ N37 in the hydrolysis of BWX, WAX and barley $\beta$-glucan respectively. * indicates $\mathrm{P}<0.05$, **** indicates $\mathrm{P}<0.0001$. (D) The hydrogen bonding interaction between the N-terminal and GH10 domains. The red dashed line indicates hydrogen bonding interaction. (E) The hydrogen bonding interaction between the C-terminal and GH10 domains. The red dotted line represents hydrogen bonds. The criterion for hydrogen bond judgment is that the distance between the acceptor and donor atoms should be less than $4 \AA$. 


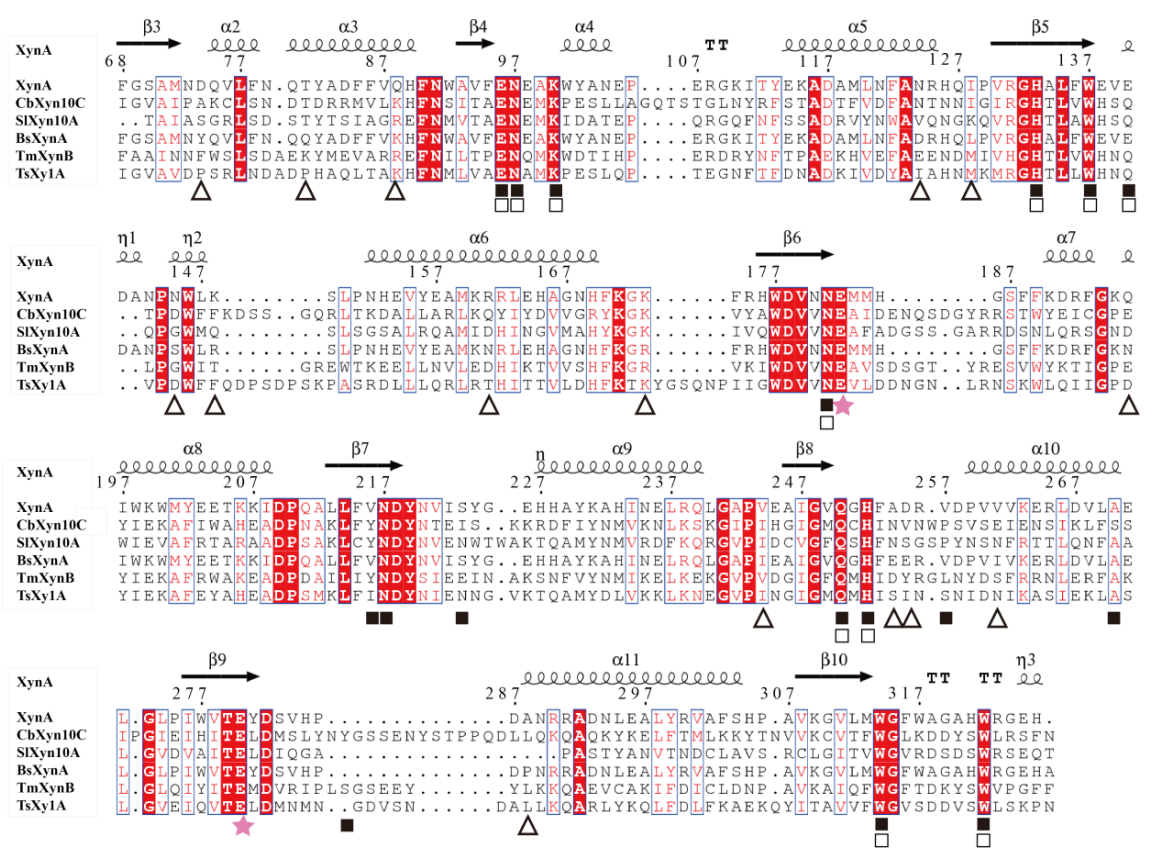

Figure 3. Multiple sequence alignment and structure analyses of XynA. Multiple sequence alignment of XynA (QCO69162) with CbXyn10C (ACM60945) from C. bescii (Chu et al., 2017); SIXyn10A (AAC26525) from S. lividans (Ducros et al., 2000b); BsXynA (AHH02587) fromBacillus sp.(Zhou et al., 2014b); TmXynB (AAD35164) from T. maritima (Ihsanawati et al., 2005); and TsXy1A (AFK86466) from T. saccharolyticum (Han et al., 2013). The red background color represents strictly conserved residues, and the red font represents the substitution of conserved residues. The pink five-pointed star indicates the two key catalytic residues of XynA, and the original secondary structure of XynA is shown above the sequence alignment. The black squares represent the residues that interact with the substrate in CbXyn10C, and the white squares represent the residues that interact with the substrate in TsXy1A. The triangles indicate the residues of XynA and BsXynA that differ in the GH10 domain. 
A

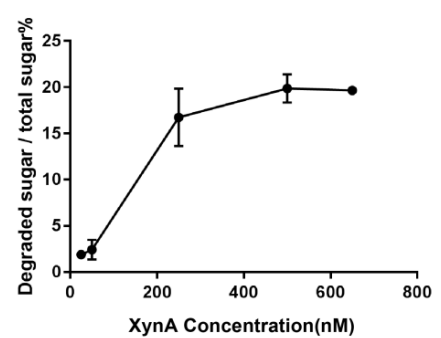

C
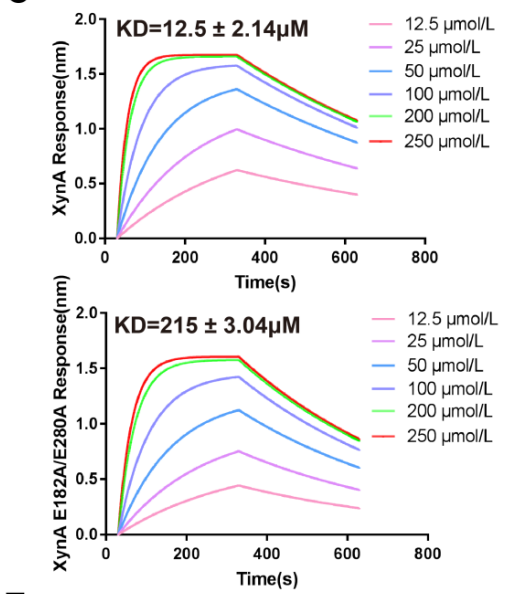

E

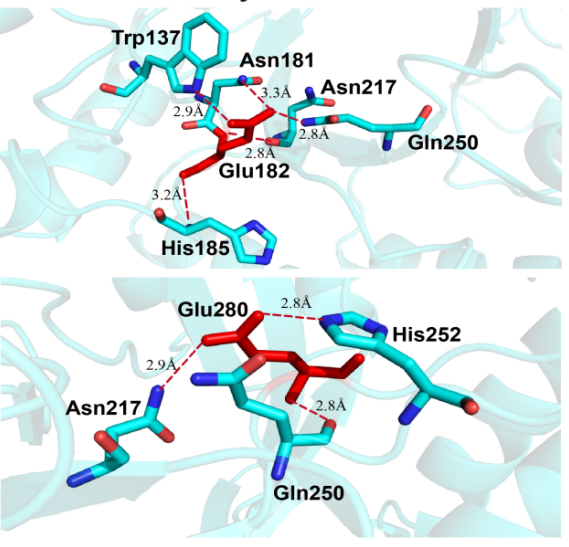

B

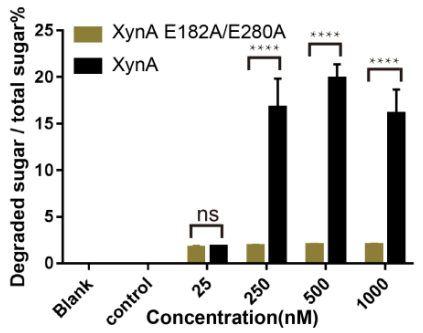

D

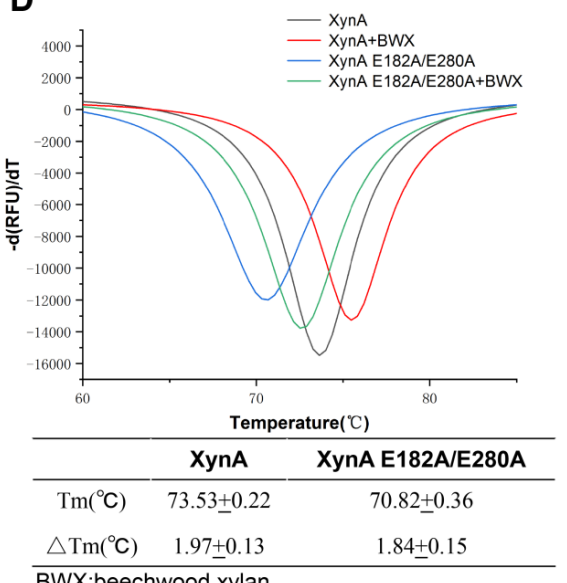

BWX:beechwood xylan

$\mathbf{F}$

XYNA E182A/E280A

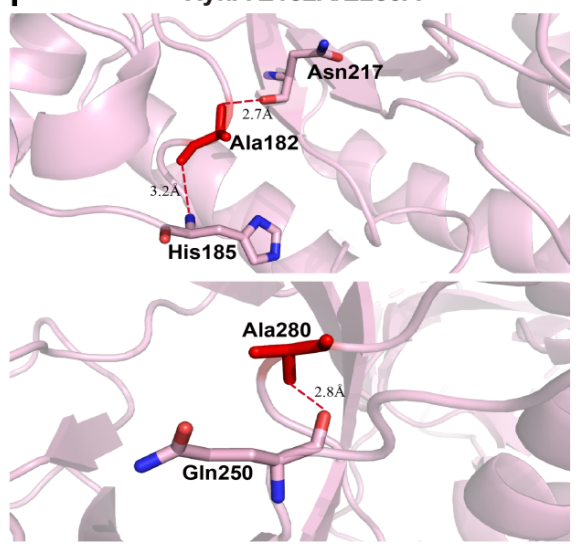

Figure 4. Comparison of XynA with XynA E182A/E280A in terms of binding, catalysis activity and structures. (A) Effect of XynA concentration on its xylanase activity. (B) XynA and XynA E182A/E280A activities for degrading substrates at different concentrations. Each experiment was repeated three times. XynA and XynA E182A/E280A were analyzed for significance, ns means no significant difference, ${ }^{*}$ means $\mathrm{P}$ $<0.05$, ${ }^{*}$ means $\mathrm{P}<0.01, * * *$ means $\mathrm{P}<0.0002$, and $* * * *$ means $\mathrm{P}<0.0001$. (C) XynA binding experiment with BWX; XynA E182A/E280A binding experiment with BWX. Different colored lines in the figure represent different concentrations of BWX. The binding constants are shown in the table. (D) Protein thermal shift assay of XynA and XynA E182A/E280A. RFU of the Y-axis represents relative fluorescence units. The Tm value represents the denaturation or exposure temperature of the hydrophobic residues of the protein. $\Delta \mathrm{Tm}$ indicates the temperature change after adding BWX. (E) Interaction between two residues Glu182 and Glu280 and surrounding residues of XynA. (F) The interaction between the Ala182 and Ala280 residues and the surrounding residues of XynA E182A/E280A. Color cyan and pink indicate the crystal structure of WT and double mutant respectively. The red dotted line represents hydrogen bonds. The criterion for 
hydrogen bond judgment is that the distance between the acceptor and donor atoms should be less than 4 $\AA$.

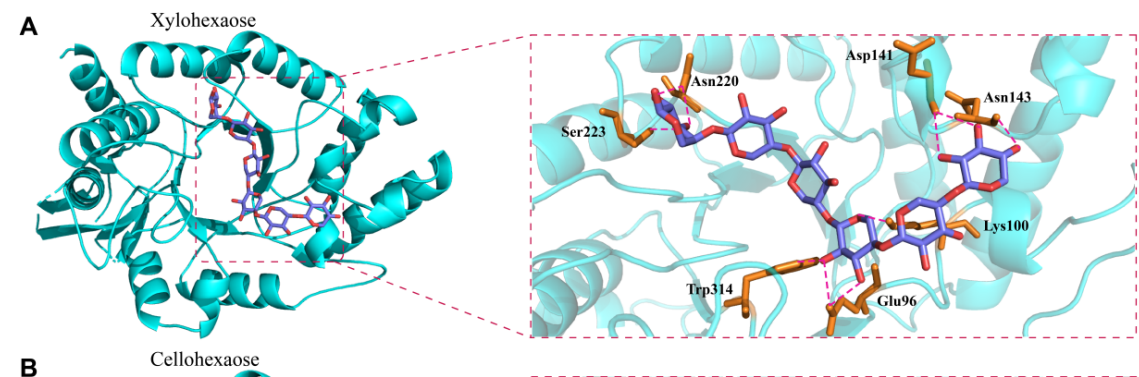

B

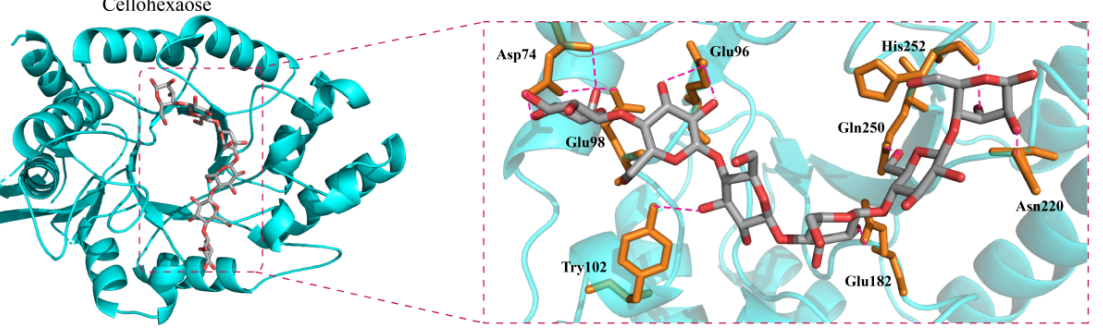

C

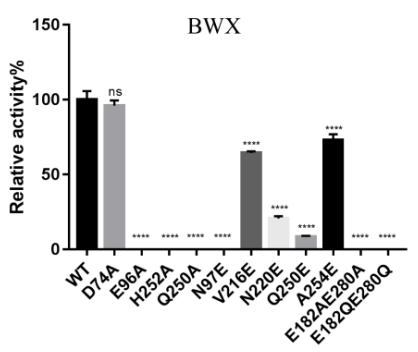

$\mathbf{E}$

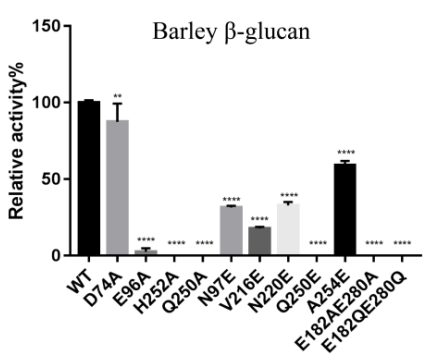

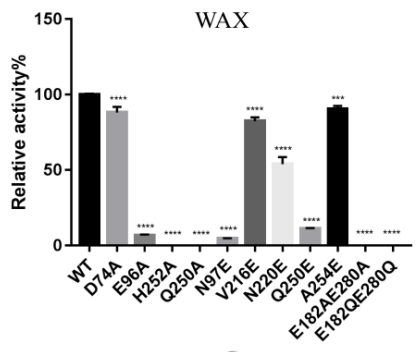

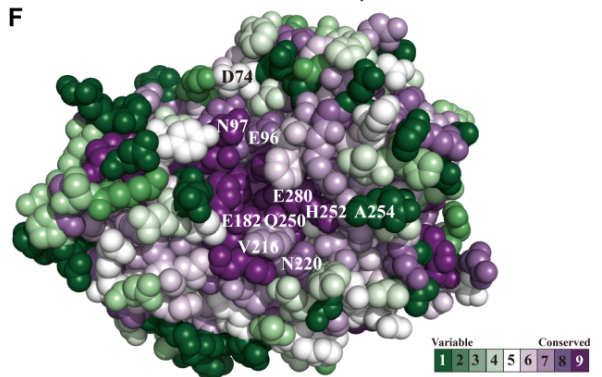

Figure 5.The molecular docking with xylohexaose and cellohexaose and activity analysis of the XynA. (A-B) The docking of XynA with xylohexaose and cellohexaose displayed in a cartoon mode, respectively, and the hydrogen bonds between the XynA with xylohexaose or cellohexaose are partially enlarged. The red dotted line indicates hydrogen bonding interaction. The criterion for hydrogen bond judgment is that the distance between the acceptor and donor atoms should be less than $3 \AA$. (C-E) Relative activities of the XynA mutants in the hydrolysis of BWX, WAX and barley $\beta$-glucan respectively. Each experiment was repeated three times. WT enzyme activity was set to $100 \%$. WT group was used as a control for a significant analysis, ${ }^{*}$ means $\mathrm{P}<0.05,{ }^{* *}$ means $\mathrm{P}<0.01,{ }^{* * *}$ means $\mathrm{P}<0.0002$, and ${ }^{* * * *}$ means $\mathrm{P}<0.0001$. (F) The spatial position and conservation of residues in space fill model of XynA that may interact with the substrate. The green to purple gradient indicates the degree of conservation. The annotated residues are the mutation sites found by molecular docking. 


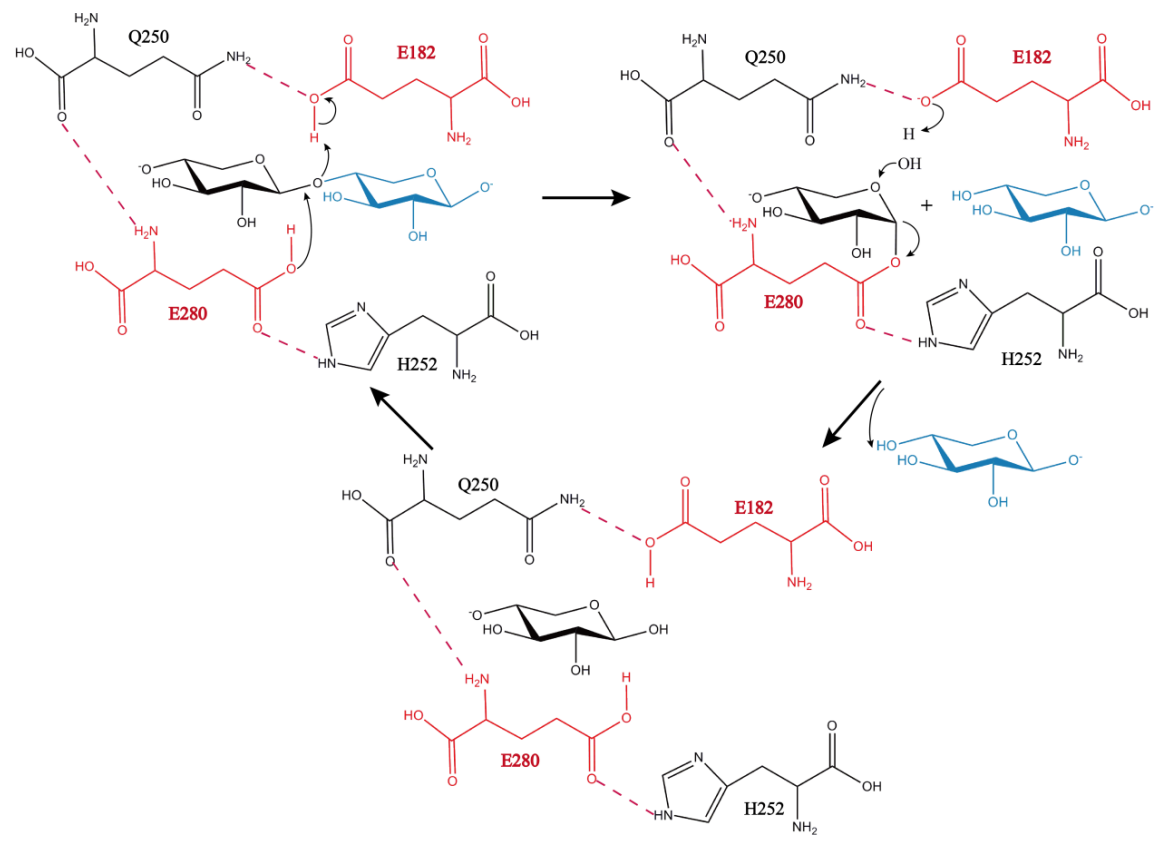

Figure 6.Proposed endo-1,4- $\beta$-xylanase catalytic mechanism for XynA. E280 acts as a nucleophile, and E182 acts as an acid/base catalyst. Q250 and H252 will interact with catalytic residues. The blue sugar represents the sugar that has been hydrolyzed. The pink dotted line represents hydrogen bond interaction.

\section{References}

Baumann, M. J., Murphy, L., Lei, N., Krogh, K. B., Borch, K., \& Westh, P. (2011). Advantages of isothermal titration calorimetry for xylanase kinetics in comparison to chemical-reducing-end assays. Anal Biochem, 410 (1), 19-26. doi:10.1016/j.ab.2010.11.001

Biely, P., Singh, S., \& Puchart, V. (2016). Towards enzymatic breakdown of complex plant xylan structures: State of the art. Biotechnol Adv, 34 (7), 1260-1274. doi:10.1016/j.biotechadv.2016.09.001

Broeker, J., Mechelke, M., Baudrexl, M., Mennerich, D., Hornburg, D., Mann, M., . . . Zverlov, V. V. (2018). The hemicellulose-degrading enzyme system of the thermophilic bacterium Clostridium stercorarium: comparative characterisation and addition of new hemicellulolytic glycoside hydrolases. Biotechnol Biofuels, 11 , 229. doi:10.1186/s13068-018-1228-3

Chakdar, H., Kumar, M., Pandiyan, K., Singh, A., Nanjappan, K., Kashyap, P. L., \& Srivastava, A. K. (2016). Bacterial xylanases: biology to biotechnology. 3 Biotech, 6 (2), 150. doi:10.1007/s13205-016-0457-z

Chu, Y., Tu, T., Penttinen, L., Xue, X., Wang, X., Yi, Z., . . . Su, X. (2017). Insights into the roles of non-catalytic residues in the active site of a GH10 xylanase with activity on cellulose. J Biol Chem, 292 (47), 19315-19327. doi:10.1074/jbc.M117.807768

Collins, T., Gerday, C., \& Feller, G. (2005). Xylanases, xylanase families and extremophilic xylanases. FEMS Microbiology Reviews, 29 (1), 3-23. doi:10.1016/j.femsre.2004.06.005

Derewenda, U., Swenson, L., Green, R., Wei, Y., Morosoli, R., Shareck, F., . . . Derewenda, Z. S. (1994). Crystal structure, at 2.6-A resolution, of the Streptomyces lividans xylanase A, a member of the F family of beta-1,4-D-glycanases. J Biol Chem, 269 (33), 20811-20814.

Dhiman, S. S., Garg, G., Sharma, J., Kalia, V. C., Kang, Y. C., \& Lee, J. K. (2014). Reduction in acute ecotoxicity of paper mill effluent by sequential application of xylanase and laccase. PLoS One, 9 (7), e102581. doi:10.1371/journal.pone.0102581 
Dodd, D., \& Cann, I. K. (2009). Enzymatic deconstruction of xylan for biofuel production. Glob Change Biol Bioenergy, 1 (1), 2-17. doi:10.1111/j.1757-1707.2009.01004.x

Dornez, E., Verjans, P., Arnaut, F., Delcour, J. A., \& Courtin, C. M. (2011). Use of psychrophilic xylanases provides insight into the xylanase functionality in bread making. J Agric Food Chem, 59 (17), 9553-9562. doi:10.1021/jf201752g

Ducros, V., Charnock, S. J., Derewenda, U., Derewenda, Z. S., Dauter, Z., Dupont, C., . . . Davies, G. J. (2000a). Substrate specificity in glycoside hydrolase family 10. Structural and kinetic analysis of the Streptomyces lividans xylanase 10A. J Biol Chem, 275 (30), 23020-23026. doi:10.1074/jbc.275.30.23020

Ducros, V., Charnock, S. J., Derewenda, U., Derewenda, Z. S., Dauter, Z., Dupont, C., . . . Davies, G. J. (2000b). Substrate specificity in glycoside hydrolase family 10. Structural and kinetic analysis of the Streptomyces lividans xylanase 10A. J Biol Chem, 275 (30), 23020-23026. doi:10.1074/jbc.275.30.23020

Emsley, P., Lohkamp, B., Scott, W. G., \& Cowtan, K. (2010). Features and development of Coot. Acta Crystallogr D Biol Crystallogr, 66 (Pt 4), 486-501. doi:10.1107/S0907444910007493

Fredriksen, L., Stokke, R., Jensen, M. S., Westereng, B., Jameson, J. K., Steen, I. H., \& Eijsink, V. G. H. (2019). Discovery of a Thermostable GH10 Xylanase with Broad Substrate Specificity from the Arctic Mid-Ocean Ridge Vent System. Appl Environ Microbiol, 85 (6), e02970-02918. doi:10.1128/aem.02970-18

Friesner, R. A., Banks, J. L., Murphy, R. B., Halgren, T. A., Klicic, J. J., Mainz,, D. T., R., . . . Perry, J. K., Shaw, D. E., Francis, P., \& Shenkin, P. S. . (2004). Glide: a new approach for rapid, accurate docking and scoring. 1. Method and assessment of docking accuracy. Journal of medicinal chemistry, 47 (7), 1739-1749. doi:10.1021/jm0306430

Gloster, T. M., Williams, S. J., Roberts, S., Tarling, C. A., Wicki, J., Withers, S. G., \& Davies, G. J. (2004). Atomic resolution analyses of the binding of xylobiose-derived deoxynojirimycin and isofagomine to xylanase Xyn10A. Chem Commun (Camb) (16), 1794-1795. doi:10.1039/b405152a

Han, X., Gao, J., Shang, N., Huang, C. H., Ko, T. P., Chen, C. C., . . . Ma, Y. (2013). Structural and functional analyses of catalytic domain of GH10 xylanase from Thermoanaerobacterium saccharolyticum JW/SL-YS485.Proteins, 81 (7), 1256-1265. doi:10.1002/prot.24286

Ihsanawati, Kumasaka, T., Kaneko, T., Morokuma, C., Yatsunami, R., Sato, T., . . . Tanaka, N. (2005). Structural basis of the substrate subsite and the highly thermal stability of xylanase 10B from Thermotoga maritima MSB8. Proteins, 61 (4), 999-1009. doi:10.1002/prot.20700

Isikgor, F. H., \& Becer, C. R. (2015). Lignocellulosic biomass: a sustainable platform for the production of bio-based chemicals and polymers. Polymer Chemistry, 6 (25), 4497-4559. doi:10.1039/c5py00263j

Ito, T., Sato, A., Takahashi, I., Ito, T., Takano, K., Noge, K., . . . Hashizume, K. (2019). Identification of enzymes from genus Trichoderma that can accelerate formation of ferulic acid and ethyl ferulate in collaboration with rice koji enzyme in sake mash. Journal of Bioscience and Bioengineering, 128 (2), 177182. doi:10.1016/j.jbiosc.2019.01.014

Keegstra, K. (2010). Plant cell walls. Plant Physiol, 154 (2), 483-486. doi:10.1104/pp.110.161240

Kelley, L. A., Mezulis, S., Yates, C. M., Wass, M. N., \& Sternberg, M. J. (2015). The Phyre2 web portal for protein modeling, prediction and analysis. Nat Protoc, 10 (6), 845-858. doi:10.1038/nprot.2015.053

Kumar, V., Marin-Navarro, J., \& Shukla, P. (2016). Thermostable microbial xylanases for pulp and paper industries: trends, applications and further perspectives. World J Microbiol Biotechnol, 32 (2), 34. doi:10.1007/s11274-015-2005-0

Laemmli, U. K. (1970). Cleavage of structural proteins during the assembly of the head of bacteriophage T4. Nature, 227 (5259), 680-685. doi:10.1038/227680a0 
Li, R., Tian, J. Z., Wang, M. R., Zhu, L. N., \& Sun, J. S. (2017). EsGLUT4 and CHHBP are involved in the regulation of glucose homeostasis in the crustacean Eriocheir sinensis. Biol Open, 6 (9), 1279-1289. doi:10.1242/bio.027532

Lisov, A. V., Belova, O. V., Lisova, Z. A., Vinokurova, N. G., Nagel, A. S., Andreeva-Kovalevskaya, Z. I., . . . Leontievsky, A. A. (2017). Xylanases of Cellulomonas flavigena: expression, biochemical characterization, and biotechnological potential. AMB Express, 7 (1), 5. doi:10.1186/s13568-016-0308-7

Liu, J., Zhang, C., \& Xu, D. (2012). QM/MM study of catalytic mechanism of Xylanase Cex from Cellulomonas fimi. J Mol Graph Model, 37 ,67-76. doi:10.1016/j.jmgm.2012.04.005

Lombard, V., Golaconda Ramulu, H., Drula, E., Coutinho, P. M., \& Henrissat, B. (2014). The carbohydrate-active enzymes database (CAZy) in 2013. Nucleic Acids Res, 42 (Database issue), D490D495. doi:10.1093/nar/gkt1178

Long, L., Tian, D., Zhai, R., Li, X., Zhang, Y., Hu, J., . . . Saddler, J. (2018). Thermostable xylanaseaided two-stage hydrolysis approach enhances sugar release of pretreated lignocellulosic biomass.Bioresour Technol, 257 , 334-338. doi:10.1016/j.biortech.2018.02.104

Mahanta, P., Bhardwaj, A., Kumar, K., Reddy, V. S., \& Ramakumar, S. (2015). Structural insights into $\mathrm{N}$-terminal to C-terminal interactions and implications for thermostability of a $(\beta / \alpha) 8$-triosephosphate isomerase barrel enzyme. Febs j, 282 (18), 3543-3555. doi:10.1111/febs.13355

McCoy, A. J., Grosse-Kunstleve, R. W., Adams, P. D., Winn, M. D., Storoni, L. C., \& Read, R. J. (2007). Phaser crystallographic software.J Appl Crystallogr, 40 (Pt 4), 658-674. doi:10.1107/S0021889807021206

Niesen, F. H., Berglund, H., \& Vedadi, M. (2007). The use of differential scanning fluorimetry to detect ligand interactions that promote protein stability. Nat Protoc, 2 (9), 2212-2221. doi:10.1038/nprot.2007.321

Otwinowski, Z., \& Minor, W. (1997). Processing of X-ray diffraction data collected in oscillation mode. Methods in enzymology, $276,307-326$.

Petersen, R. L. (2017). Strategies Using Bio-Layer Interferometry Biosensor Technology for Vaccine Research and Development.Biosensors (Basel), 7 (4). doi:10.3390/bios7040049

Sastry, G. M., Adzhigirey, M., Day, T., Annabhimoju, R., \& Sherman, W. (2013). Protein and ligand preparation: parameters, protocols, and influence on virtual screening enrichments. Journal of computeraided molecular design, 27 (3), 221-234. doi:10.1007/s10822-013-9644-8)

Sims, R. E., Mabee, W., Saddler, J. N., \& Taylor, M. (2010). An overview of second generation biofuel technologies. Bioresour Technol, 101 (6), 1570-1580. doi:10.1016/j.biortech.2009.11.046

Song, L., Tsang, A., \& Sylvestre, M. (2015). Engineering a thermostable fungal GH10 xylanase, importance of N-terminal amino acids.Biotechnol Bioeng, 112 (6), 1081-1091. doi:10.1002/bit.25533

V Ducros, S J Charnock, U Derewenda, Z S Derewenda, Z Dauter, C Dupont, . . . Davies., G. J. (2000). Substrate Specificity in Glycoside Hydrolase Family 10. Structural and Kinetic Analysis of the Streptomyces Lividans Xylanase 10A. Journal of Biological Chemistry, 275 (30), 23020-23026. doi:10.1074/jbc.275.30.23020

Victor, D. G., \& Leape, J. P. (2015). Global climate agreement: After the talks. Nature, 527 (7579), 439-441. doi:10.1038/527439a

Wang, K., Cao, R., Wang, M., Lin, Q., Zhan, R., Xu, H., \& Wang, S. (2019). A novel thermostable GH10 xylanase with activities on a wide variety of cellulosic substrates from a xylanolytic Bacillus strain exhibiting significant synergy with commercial Celluclast $1.5 \mathrm{~L}$ in pretreated corn stover hydrolysis. Biotechnol Biofuels, 12 , 48. doi:10.1186/s13068-019-1389-8

Withers, S., Dombroski, D., Berven, L., Kilburn, D., Miller, R., Warren, R., \& Gilkes, N. (1986). Direct 1H N.M.R. determination of the stereochemical course of hydrolyses catalysed by glucanase components of the 
cellulase complex. Biochemical and biophysical research communications, 139 , 487-494. doi:10.1016/S0006$291 X(86) 80017-1$

Xiaoyun Su, Yejun Han, Dylan Dodd, Young Hwan Moon, Shosuke Yoshida, Roderick I Mackie, \& Cann, I. K. O. ( 2013). Reconstitution of a Thermostable Xylan-Degrading Enzyme Mixture from the Bacterium Caldicellulosiruptor bescii. Appl Environ Microbiol, 79 (5), 1481-1490. doi:10.1128/AEM.03265-12

You, S., Xie, C., Ma, R., Huang, H. Q., Herman, R. A., Su, X. Y., . . . Luo, H. Y. (2019). Improvement in catalytic activity and thermostability of a GH10 xylanase and its synergistic degradation of biomass with cellulase. Biotechnol Biofuels, 12 , 278. doi:10.1186/s13068-019-1620-7

Zhang, Y., An, J., Yang, G., Zhang, X., Xie, Y., Chen, L., \& Feng, Y. (2016). Structure features of GH10 xylanase from Caldicellulosiruptor bescii: implication for its thermophilic adaption and substrate binding preference. Acta Biochim Biophys Sin (Shanghai), 48 (10), 948-957. doi:10.1093/abbs/gmw086

Zheng, F., Huang, J., Liu, X., Hu, H., Long, L., Chen, K., \& Ding, S. (2016). N- and C-terminal truncations of a GH10 xylanase significantly increase its activity and thermostability but decrease its SDS resistance. Appl Microbiol Biotechnol, 100 (8), 3555-3565. doi:10.1007/s00253-015-7176-y

Zhou, J., Wu, Q., Zhang, R., Mo, M., Tang, X., Li, J., . . . Huang, Z. (2014a). A thermo-halo-tolerant and proteinase-resistant endoxylanase from Bacillus sp. HJ14. Folia Microbiol (Praha), 59 (5), 423-431. doi:10.1007/s12223-014-0316-4

Zhou, J., Wu, Q., Zhang, R., Mo, M., Tang, X., Li, J., . . . Huang, Z. (2014b). A thermo-halo-tolerant and proteinase-resistant endoxylanase from Bacillus sp. HJ14. Folia Microbiol (Praha), 59 (5), 423-431. doi:10.1007/s12223-014-0316-4 